\title{
Novedosa herramienta didáctica para la conversión de tasas de interés financiero
}

\section{Novel teaching tool for the conversion of financial interest rates}

Milton-Hernando Triana-Lozano ${ }^{1}$

\section{Palabras clave: \\ Interés, Tasa de interés, Enseñanza, \\ Aprendizaje, \\ Operaciones bancarias}

Artículo de investigación:

Fecha de recepción:

2019/11/04

Fecha de aceptación:

2019/12/16

Esta publicación se encuentra bajo licencia:

Creative Commons ReconocimientoNoComercialSinObraDerivada 4.0 Internacional

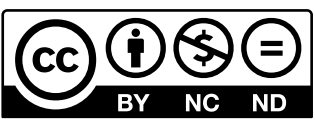

\section{Resumen}

La conversión de tasas o equivalencia de tasas de interés es la base inicial y fundamental para la adecuada comprensión de los temas de matemáticas financieras.

Los métodos tradicionales son todos eficaces (consiguen su objetivo), pero al mismo tiempo son bien complejos y muchas veces no son tan eficientes en el proceso de enseñanza-aprendizaje.

Se construyó una herramienta, denominada la "Matriz MILHER", que facilita la labor de conversión de tasas por su practicidad y eficiencia, para lo cual se cursó una fase experimental inicial y se acudió al método cuantitativo, realizando planteamientos con ejercicios prácticos claves, tanto a docentes como a estudiantes de matemáticas financieras.

Los resultados han sido positivos, ya que la "Matriz MILHER" contrastada con los métodos tradiciones en términos de practicidad es menos compleja y más amigable en su uso, y en términos de eficiencia facilita un ahorro del recurso tiempo bien interesante, logrando idénticos y similares resultados que con los métodos tradicionales empleados para el mismo fin.

1 Contador Público Certificado, profesor de la Universidad Santo Tomás sede Villavicencio (Meta) Colombia, orcid.org/000-0001-6332-0173. miltontriana@ usantotomas.edu.co, código ORCID: https://orcid.org/0000-0001-6332-0173 


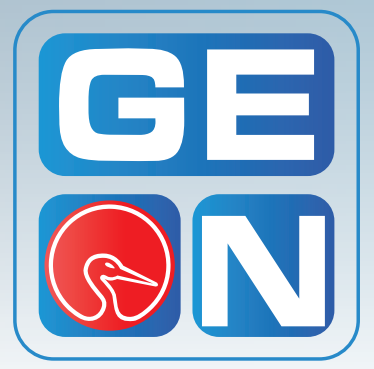

\section{Revista GEON}

(Gestión, Organizaciones y Negocios)

ISSN: 2346-3910 en línea

revistageon@unillanos.edu.co

Universidad de los Llanos

Colombia

\section{Triana Lozano, M. (2020).}

Novedosa herramienta didáctica para la conversión de tasas de interés financiero.

\section{Revista GEON}

(Gestión, Organizaciones y Negocios), 7(1), 49-69.

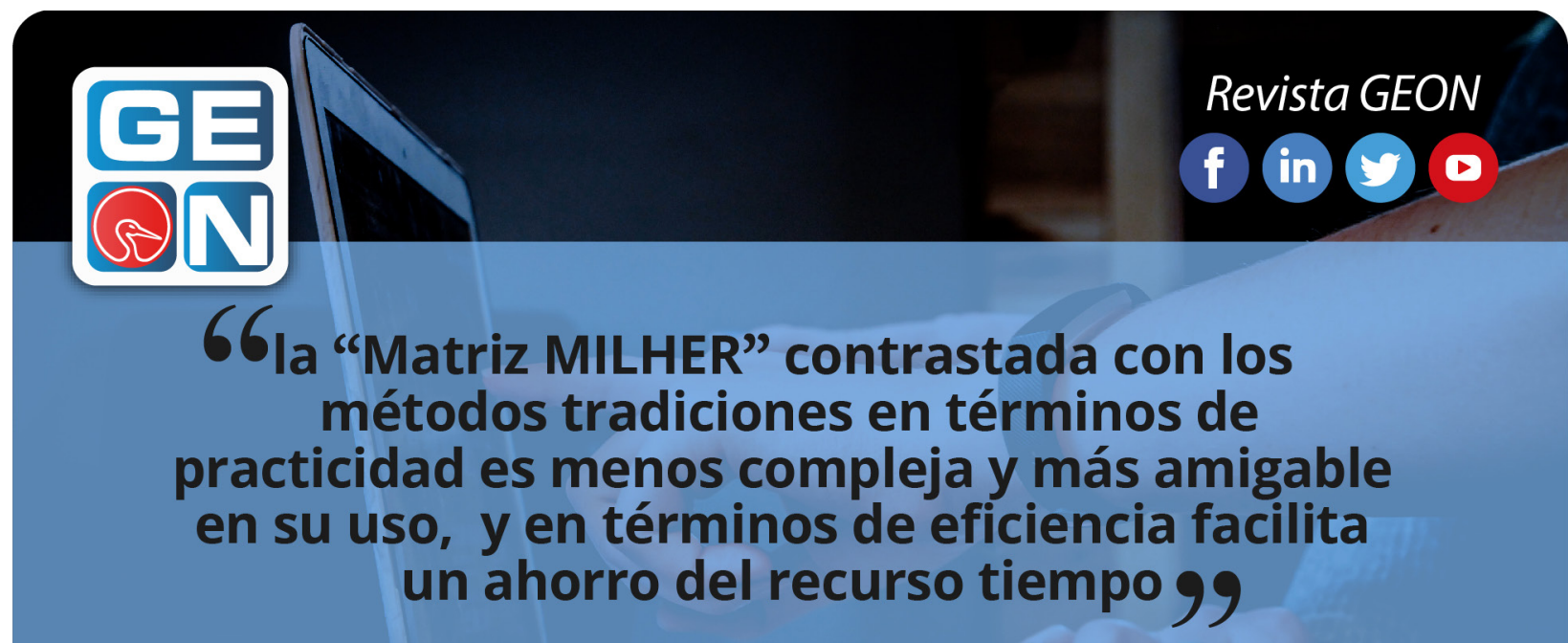

Triana Lozano, M. (2020). Novedosa

herramienta didáctica para la conversión de tasas de interés financiero. Revista GEON (Gestión, Organizaciones Y Negocios), 7(1), 49-69. 
Se espera poder seguir replicando esta novedosa herramienta educativa con la comunidad académica universitaria dentro y fuera del país.

Palabras clave: Interés, Tasa de interés, Enseñanza, Aprendizaje, Operaciones bancarias

\section{Abstract}

The conversion of rates or equivalence of interest rates is the initial and fundamental basis for the proper understanding of the topics of financial mathematics.

Traditional methods are all effective (they achieve their objective), but at the same time they are very complex and often not as efficient in the teaching-learning process.

Initially, it was necessary to carry out a documentary work with multiple bibliographic consultations to understand the structure and operation of the traditional methods for the conversion of rates.

A tool, called the "MILHER Matrix" was built, which facilitates the work of conversion of rates for its practicality and efficiency, for which an initial experimental phase was taken and the quantitative method was used, making approchaes with key practical exercises, both teachers and students of financial mathematics.

The results have been positive, since the "MILHER Matrix" contrasted with the traditional methods in terms of practicality is less complex and friendlier in its use, and in terms of efficiency it facilitates a very interesting time saving of the resource, achieving identical and similar results that with the traditional methods used for the same purpose.

It is expected to continue applying this innovative educational tool with the university academic community inside and outside the country.

Keywords: Interest, Interest rate, Teaching, Learning, Banking operations 


\section{Introducción}

Hay que comprender inicialmente que la conversión de tasas de interés financiero es un conocimiento indispensable para el dominio de los principales temas en la asignatura de matemáticas financieras, como anualidades, gradientes, indicadores de evaluación financiera de proyectos, entre otros; cabe recordar que "las matemáticas financieras son fundamentales para la adecuada toma de decisiones. financieras." (Agudelo Fernández, 2019, pág. 22)

Se conoce, también, que hay reconocidos procedimientos que procuran el dominio de este importante tema, como lo es la "Gráfica de equivalencias de tasas" de Baca Currea, el método "Con base en preguntas" de Ramírez y Martínez, el "Diagrama de conversión de tasas de interés" de Moreno y Rueda, el de "Transformación de tasas de interés" de Vélez, el de la "Gráfica de conversión de tasas" de Meza Orozco, entre otros, que son eficaces pues cumplen su objetivo, pero que son complejos en su uso y no muy eficientes en el manejo del recurso tiempo.

Por lo tanto, como resultado del ejercicio docente con estudiantes universitarios de Villavicencio (Meta) en el proceso de enseñanza aprendizaje de las matemáticas financieras y teniendo en cuenta que se debe favorecer e "implementar programas que fomenten una cultura financiera perspicaz" (Pérez Paredes, Cruz de los Ángeles, \& Gómez Pulido, 2018, pág. 124), se acuñó una herramienta que supera las dificultades anteriores, que logra similares e idénticos resultados, que simplifica el proceso realizado y que a su vez optimiza el manejo de los recursos empleados, principalmente el tiempo.

Se enunciaron algunos conceptos indispensables en el manejo del dinero durante el tiempo, como las tasas de interés, el reconocimiento de sus componentes (clase, periodicidad y modalidad) y los procedimientos tradicionales para la conversión de tasas o equivalencia de tasas; seguidamente, se expuso los materiales y métodos que facilitaron lograr los objetivos específicos planteados, y finalmente se presentó el nuevo tema de discusión: la propuesta de una herramienta para la conversión de tasas que simplifica el proceso y economiza el tiempo de uso, denominada la

\section{"Matriz MILHER".}

\section{Objetivos}

El objetivo general planteado fue el "presentar una propuesta de herramienta didáctica que facilite la conversión de tasas de interés financiero y que optimice su proceso" para lo cual se procuró, por un lado, "explicar la estructura y funcionamiento de la novedosa herramienta" $y$, por otro lado, "contrastar el uso de otros procedimientos tradicionales con la Matriz MILHER para la conversión de tasas de interés financiero."

\section{Contexto teórico}

\section{El interés}

La avaricia y el interés eran condenados en el antiguo testamento de la 
Biblia. Algunos filósofos griegos también desaprobaban el préstamo con intereses y en la Edad Media también se mantuvo el criterio de que el dinero no podía engendrar dinero. (Carrasco Manchado \& Rábade Obradó, 2008, pág. 193)

En la Edad Moderna se menguó dicha posición, al "estipularse un interés en el préstamo de cosas que se consumen por el uso" (Jiménez Muñoz, 2010, pág. 42) y hoy ya es común su cobro, por lo que "el interés está involucrado en la mayoría de operaciones comerciales" (Frare, 2005, pág. 82).

Ahora bien, "el concepto del valor del dinero en el tiempo, se sustenta en el hecho de que el dinero disponible ahora, vale más que la expectativa de la misma cantidad en unperiodo futuro" (Lezama Vásquez, 2006); es decir, el valor del dinero en el tiempo, es el "valor recibido o entregado por el uso del dinero, utilidad o ganancia que genera un capital, el precio que se paga por el uso del dinero, rendimiento de una inversión" (Morales Castaño, 2014, pág. 19).

\section{Las operaciones financieras}

La operación financiera es "toda acción que intercambia o sustituye unos capitales financieros por otros de distinto vencimiento, mediante la aplicación de una determinada ley financiera en un punto de referencia". (Mira Navarro, 2019)

Ahora bien, se llama "capitalización a la operación financiera que permite obtener el valor futuro de un capital ubicado en un momento determinado del tiempo"(Margaria \& Bravino, 2014, pág. 26) y "actualización a la operación financiera que consiste en obtener el valor actual de un capital futuro" (Margaria \& Bravino, 2014, pág. 28).

\section{La tasa de interés}

Inicialmente hay que resaltar que el interés "se expresa en monto o en porcentaje del capital negociado por período. Como importe de alquiler que es, el interés debe referirse a períodos de tiempo y, según el capital comprometido, se puede establecer el monto periódico o el porcentaje correspondiente" (Buenaventura Vera, 2018, pág. 2) y es esto a lo que se denomina tasa de interés.

La tasa de interés se puede expresar de dos maneras: en factor decimal (forma algebráica), que es la que se utiliza en los ejercicios, y en porcentaje que es la que se maneja verbalmente. (Diaz Mata \& Aguilera Gómez, 2013, pág. 36)

\section{Interés simple e interés compuesto}

Cabe expresar que el interés simple "es la manera de calcular el interés en la que el capital de la inversión o crédito no se incrementa con los intereses causados periódicamente. Así, en el interés simple los intereses se calculan periódicamente sobre la misma base de capital, claro está, siempre que no se hagan adiciones o retiros." (Agudelo Fernández, 2019, pág. 50)

Por otro lado, el interés compuesto es "una modalidad financiera, que acumula el capital, el interés generado en un 
periodo de tiempo, formando de esta manera, en cada periodo, un nuevo capital. Y, sobre este valor, se calculan los intereses para el siguiente periodo." (Herrera Aráuz, 2017, pág. 44)

\section{Elementos de la tasa de interés}

Es importante decir que cuando se reconoce una tasa de interés hay que distinguir perfectamente su clase, su periodo de aplicación, la base de aplicación y su forma de aplicación.

\section{Clases de tasas de interés}

Hay dos clases de tasas de interés compuesto: la tasa de interés nominal y la tasa de interés efectiva; la primera "es la tasa de interés que expresada anualmente capitaliza varias veces al año" (Cano Morales, 2017, pág. 89) y la segunda "es la tasa que realmente se aplica en el periodo de capitalización sobre el capital, para calcular los intereses." (Cano Morales, 2017, pág. 88)

Aquí es importante resaltar que existe un paralelo entre el interés simple y la tasa nominal, y el interés compuesto y la tasa efectiva. En las dos primeras, no se tiene en cuenta la reinversión, mientras que en las últimas sí (RosiIlo C., 2009, pág. 49), y que "las tasas efectivas son indicadores que ayudan a los inversionistas y a los asesores financieros a tomar la mejor decisión para invertir sus capitales. (Villalobos Pérez, 2017, pág. 174)

\section{Periodicidad de las tasas de interés}

La periodicidad o frecuencia de conversión o periodo de capitalización es el número de periodos que que se capitaliza el interés en un año, es decir el interés puede ser convertido en capital en forma anual, semestral, trimestral, mensual, etc. (Diaz Mata \& Aguilera Gómez, 2013, pág. 69)

\section{Modalidad de las tasas de interés}

La modalidad de las tasas de interés depende del momento de aplicación o de la forma de aplicación: con tasas de interés vencida los intereses "se pagan, adeudan o capitalizan al finalizar el periodo de liquidación" y con tasas de interés anticipada los intereses "se pagan, adeudan o capitalizan al inicio del periodo de liquidación". (Gutiérrez Carmona, 2012)

\section{Tasas de interés equivalentes}

En el entorno económico "cuando se consideran juntos, el valor del dinero en el tiempo y la tasa de interés permiten formular el concepto de equivalencia económica, que implica que dos sumas diferentes de dinero en diferentes tiempos tienen el mismo valor económico" (Blank \& Tarquin, 2006) y, consecuentemente, "dos tasas de interés son equivalentes si con diferentes periodos de capitalización producen iguales intereses en el mismo plazo (Villalobos Pérez, 2017, pág. 178), es decir, que cuando dos operaciones son equivalentes es porque sus tasas de interés también son equivalentes.

Como consecuencia conviene saber cambiar o convertir la clase de tasas entre sí (de nominal a efectiva o de efectiva a nominal), la modalidad 
de las tasas entre sí (de anticipada a vencida o de vencida a anticipada) y la periodicidad de las tasas (cualquiera que ella sea), o de uno a otro concepto si así se requiere.

\section{Procedimientos, Instrumentos y herramientas para conversión de tasas}

\section{Procedimiento 1: Gráfica de equivalencias de tasas de Baca Currea}

La Gráfica de equivalencia de tasas (Baca Currea, 2007, pág. 37) es de bastante aplicación y utilización por docentes universitarios (por esta parte de Colombia) en la enseñanza-aprendizaje de este importante tema (Figura 1), por su versatilidad y simplicidad en su manejo:

Figura 1. Gráfica de equivalencia de tasas

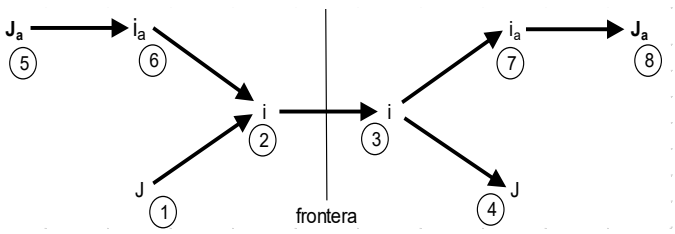

Fuente: Texto Ingeniería Económica (Baca Currea, 2007, pág. 27)

Emplea en su estructura algunas convenciones que significan lo siguiente:

$\mathbf{J}_{\mathbf{a}}$ : tasa de interés nominal anticipada.

$\mathbf{i}_{\mathbf{a}}$ : tasa de interés efectiva anticipada.

J: tasa de interés nominal vencida.

i: tasa de interés efectiva vencida.

Para el uso de la Gráfica de equivalencia de tasas, siempre se deben comenzar de un punto de la izquierda y seguir la trayectoria hasta llegar a otro punto situado en la parte de la derecha. (Baca Currea, 2007, pág. 27)

\section{Procedimiento 2: \\ Con base en preguntas de Ramírez y Martínez}

En este método parte de una igualdad entre la tasa conocida que se ubica en la parte izquierda y la tasa desconocida que se sitúa en parte izquierda (Figura 2)

Figura 2. Con base en preguntas

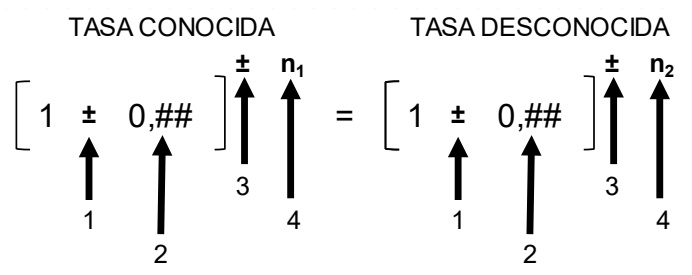

Fuente: Elaborado con base en Texto Matemática financiera (Ramírez Mora \& Martínez Cárdenas, 2016, pág. 84)

Con este método se requiere realizar cuatro preguntas consecutivas a cada lado del igual de la ecuación:

1. Cuál es la condición de la tasa (modalidad), para lo cual se ubica el signo más (+) si es vencida o el signo menos (-) si es anticipada

2. Cuál es el tipo de la tasa de interés (nominal o efectiva), ya que en el primer caso se dividirá por el número de capitalizaciones y en el segundo caso no hay denominador.

3. Cuál es el signo del exponente de la ecuación, como la primera pregunta, en donde el signo más $(+)$ es para tasa vencida o el signo menos (-) es para tasa anticipada. 
4. Cuál es el valor del exponente, como la segunda pregunta, si es nominal se ubicará el número de capitalizaciones y si es efectiva el número de la periodicidad de la tasa.

\section{Procedimiento 3: \\ Diagrama de conversión de tasas de interés de Moreno y Rueda}

Figura 3. Diagrama de conversión de tasas de interés de Moreno y Rueda

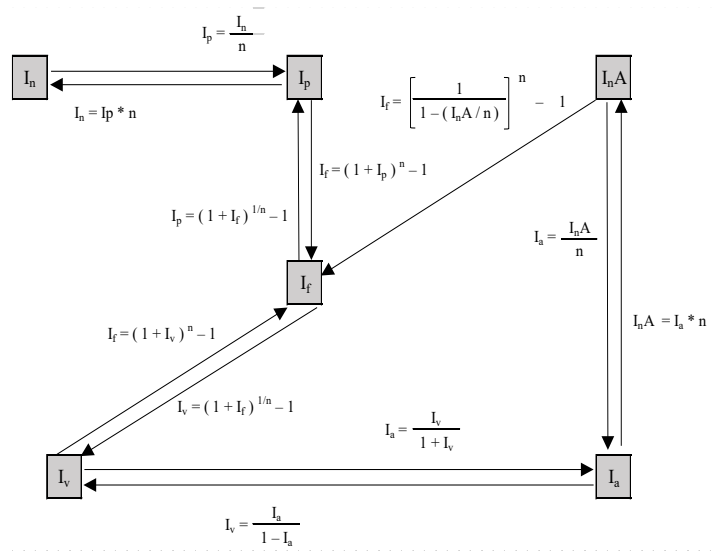

Fuente: (Moreno Gómez \& Rueda Forero, 1998, pág. 48)

Este Diagrama de conversión de tasas (Figura 3) para ser empleado requiere el empleo de estas convenciones:

$\mathbf{I}_{\mathbf{a}}$ : Interés anticipado

Iv: Interés vencido

I. Interés efectivo

$\mathbf{I}_{\mathbf{p}}$ : Interés periódico

$\mathbf{I}_{\mathbf{n}}$ : Interés nominal vencido

$\mathbf{I}_{\mathbf{n}} \mathbf{A}$ : Interés nominal anticipado

n: número de capitalizaciones o periodos

Las recomendaciones son: seguir las flechas e identificar los componen- tes de la tasa de interés (tipo o clase, unidad o periodicidad y condición o modalidad) tanto de la tasa conocida como de la desconocida; la limitante está es que se deben tener tasas con igual periodicidad para su aplicación.

\section{Procedimiento 4:}

\section{Transformación de tasas de interés de Vélez}

Figura 4. Transformación de tasas de interés de Vélez Pareja

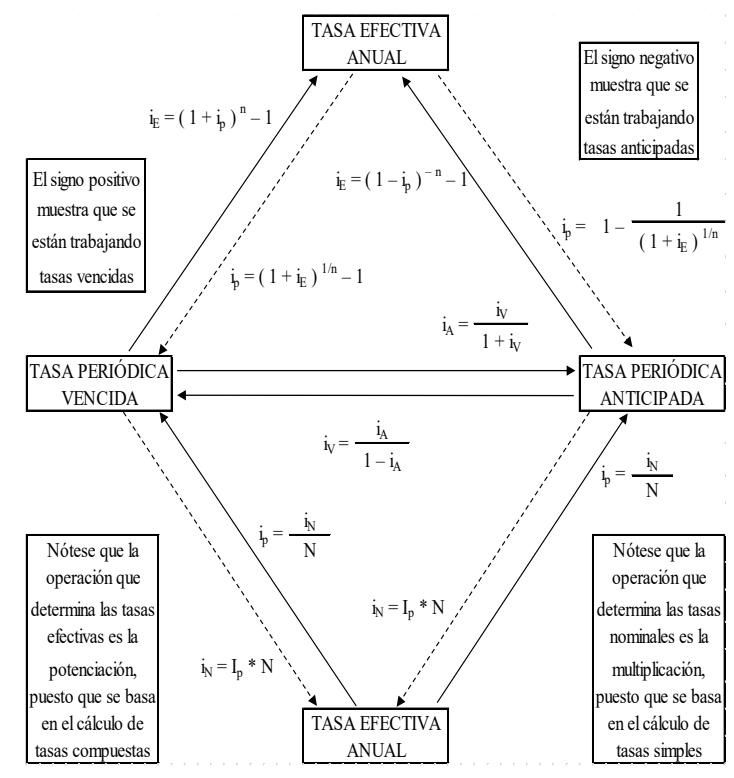

Fuente: (Vélez Pareja, 2006, pág. 119)

Este diagrama para la transformación de tasas de interés de Vélez, para ser utilizado, tiene las siguientes convenciones:

$\mathbf{i}_{\mathbf{E}}$ : tasa efectiva

$\mathbf{i}_{\mathbf{N}}$ : tasa nominal

$\mathbf{i}_{\mathbf{A}}$ : tasa periódica anticipada

$\mathbf{i}_{\mathbf{v}}$ : tasa periódica vencida

$\mathbf{i}_{\mathbf{p}}$ : tasa periódica

$\mathbf{N}$ : número de capitalizaciones o periodos 
Se deben tener en cuenta algunas recomendaciones: seguir las flechas e identificar los componentes de la tasa de interés (tipo o clase, unidad o periodicidad y condición o modalidad) tanto de la tasa conocida como de la desconocida; también el inconveniente es que se deben trabajar tasas con igual periodicidad.

\section{Procedimiento 5: \\ Gráfica de conversión de tasas de interés de Meza Orozco}

Figura 5. Diagrama de conversión de tasas de interés de Meza

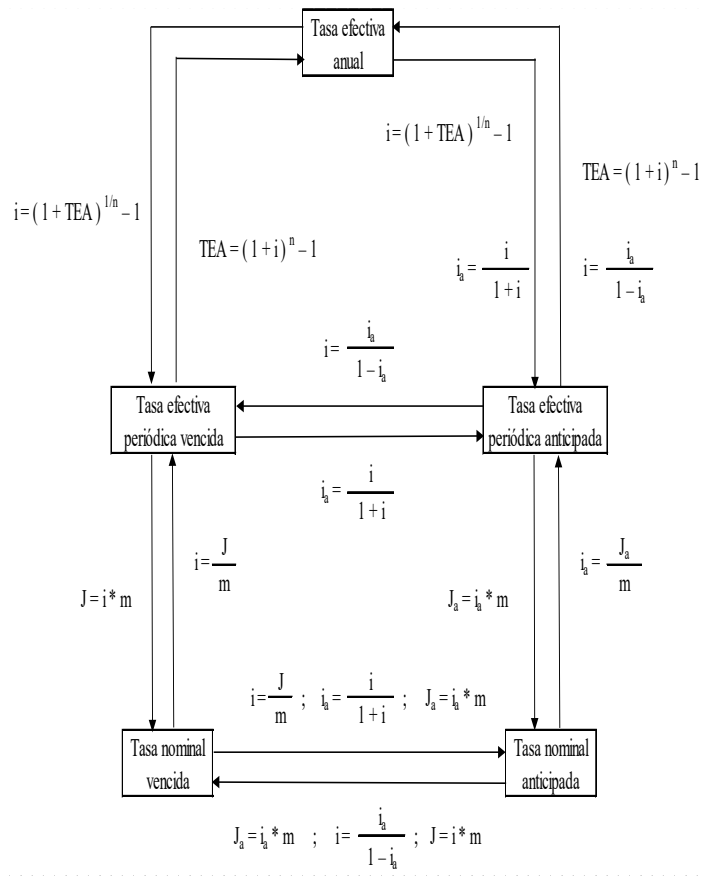

Fuente: Texto Matemática financiera. (Meza Orozco, 2017, pág. 180)

Al igual que los diagramas anteriores, se deben tener en cuenta las mismas recomendaciones: seguir las flechas e identificar los componentes de la tasa de interés (tipo o clase, unidad o periodicidad y condición o modalidad) tanto de la tasa conocida como de la desconocida; también el inconvenien- te se da en que se deben trabajar tasas con igual periodicidad

Este diagrama utiliza las siguientes convenciones:

TEA: tasa efectiva anual

$\mathbf{J}_{\mathbf{a}}$ : tasa nominal anticipada

J: tasa nominal vencida

$\mathbf{i}_{\mathbf{a}}$ : tasa periódica anticipada

i: $\quad$ tasa periódica vencida

n: periodicidad de la tasa periódica o efectiva

m: capitalizaciones de la tasa nominal

\section{Materiales y métodos}

Este trabajo por el nivel de conocimientos fue documental y experimental porque se pretendió "establecer el posible efecto de una causa que se manipula, procurando un alto grado de validez tanto interna como externa (Hernandez Sampieri, Fernández Collado, \& Baptista Lucio, 2010, pág. 130).

Se realizó una serie de consultas bibliográficas sobre el tema de conversión de tasas de interés con fuentes de textos universitarios y se indagó con docentes universitarios sobre los procedimientos de enseñanza aprendizaje para explicar el tema de conversión de tasas de interés o equivalencias de tasas.

Así pues, se tuvieron en cuenta algunas fórmulas existentes y se plantearon nuevas fórmulas para el diseño final de la "Matriz MILHER"; luego se probó la novedosa herramienta 
con estudiantes de matemática financiera de tres universidades de la región Fundación universitaria San Martín (FUSM), Corporación universitaria Minuto de Dios (UNIMINUTO) y Universidad Santo Tomás (USTA) y se sistematizaron los resultados de la aplicación inicial de la "Matriz MILHER" en comparación con otras herramientas empleadas para el mismo fin, haciendo pruebas con 6 ejercicios de base de diferente tenor, lo cual permitió evaluar la eficiencia y la aceptación de esta nueva herramienta en cuanto a su contenido y apariencia a nivel general, y en cuanto a la concordancia y la relevancia de cada ítem a nivel particular en el proceso de conversión de tasas de interés.

Se acudió al método cuantitativo, entonces, en cuanto que hubo mediciones, estuvo "orientado a la comprobación confirmatoria" y al "resultado", con "datas sólidos y repetibles" (Reichardt \& Cook, 1986), ya que se hicieron planteamientos de tipo numérico a nivel experimental, desde donde se esbozó un informe documental sobre situaciones habituales donde se precisa conocer y dominar la equivalencia de las tasas de interés financieras, que generan dudas en su aplicación.

Cabe decir que se aplicaron dos encuestas: la primera, para indagar el reconocimiento de los elementos de las tasas de interés y el procedimiento empleado para conversión de tasas y la percepción del método utilizado para ellos; y la segunda, para identificar la percepción que tuvieron los preguntados sobre el uso de la "Matriz MILHER" y su apariencia en contraste con los demás procedi- mientos empleados para la conversión de tasas.

Para lo anterior, se consideró como fuentes primarias las encuestas y los trabajos experimentales de campo, y como fuentes secundarias se empleó los contenidos de diferentes textos de matemáticas financieras y otras fuentes bibliográficas que versan sobre conversión de tasas o equivalencia de tasas de interés financiero.

\section{Resultados}

\section{Estructura de la novedosa herramienta denominada "Matriz MILHER".}

Seguidamente se presenta una novedosa herramienta para la conversión o equivalencia de tasas, que se denomina la "Matriz MILHER" en su segunda versión (Figura 6).

Esta "Matriz MILHER" mejorada consta de 4 columnas y obedece a la secuencia práctica de su utilización:

La primera columna presenta las cuatro opciones (cajas) de la tasa de interés conocida, es decir, de la que se va a convertir; pueden ser: una tasa nominal anticipada $\left(\mathbf{J}_{\mathbf{a}}\right)$, una tasa nominal vencida (J), una tasa efectiva anticipada $\left(\mathbf{i}_{\mathbf{a}}\right)$ o una tasa efectiva vencida (i).

La segunda columna precisa las cuatro opciones de destino de la tasa de interés desconocida y son cuatro renglones por cada caja de la primera columna: la tasa nominal anticipada $\left(\mathbf{J}_{\mathbf{a}}\right)$, la tasa nominal vencida (J), la tasa efectiva anticipada (i $\mathbf{i}_{\mathbf{a}}$ ) o la tasa efectiva vencida (i). 
Novedosa herramienta didáctica para la conversión de tasas de interés financiero, 4马-6马

Figura 6. La Matriz MILHER v2

\begin{tabular}{|c|c|c|c|}
\hline \multicolumn{2}{|c|}{$D E \Longrightarrow A$} & Misma periodicidad & Diferente periodicidad \\
\hline \multirow{4}{*}{$\begin{array}{c}\text { Nominal } \\
\text { Anticipada } \\
\left(\mathrm{J}_{\mathrm{a}}\right)\end{array}$} & Nominal Anticipada & & $J_{a}=\left(1-\left(1-J_{a} / m\right)^{(m / m d)}\right)^{*} m d$ \\
\hline & \begin{tabular}{|l} 
Nominal Vencida \\
\end{tabular} & $J=\left(1 /\left(1-J_{a} / m\right)-1\right)^{*} m$ & $J=\left(1 /\left(1-J_{a} / m\right)^{(m / m d)}-1\right)^{*} m d$ \\
\hline & Éfectiva Aniticipada & $i_{a}=J_{a} / m$ & $\mathrm{i}_{\mathrm{a}}=1-\left(1-\mathrm{J}_{\mathrm{a}} / \mathrm{m}\right)^{(\mathrm{m} / \mathrm{md})}$ \\
\hline & Efectiva Vencida & $\mathrm{i}=1 /\left(1-\mathrm{J}_{\mathrm{a}} / \mathrm{m}\right)-1$ & $\mathrm{i}=1 /\left(1-\mathrm{J}_{\mathrm{a}} / \mathrm{m}\right)^{(\mathrm{m} / \mathrm{md})}-1$ \\
\hline \multirow{4}{*}{$\begin{array}{c}\text { Nominal } \\
\text { Vencida } \\
\text { (J) }\end{array}$} & Nominal Anticipada & $J_{a}=(1-1 /(1+J / m))^{*} m$ & $J_{a}=\left(1-1 /(1+J / m)^{(m / m d}\right)^{*} m d$ \\
\hline & Nominal Vencida & & $J=\left((1+J . / m)^{(\mathrm{m} / \mathrm{md})}-1\right)^{*} \mathrm{md}$ \\
\hline & Efectiva Anticipada & $i_{a}=1-1 /(1+J / m)$ & $i_{a}=1-1 /(1+J / m)^{(m / m d)}$ \\
\hline & Efectiva Vencida & $\mathrm{i}=\mathrm{J} / \mathrm{m}$ & $\mathrm{i}=(1+\mathrm{J} / \mathrm{m})^{(\mathrm{m} / \mathrm{md})}-1$ \\
\hline \multirow{4}{*}{$\begin{array}{c}\text { Efectiva } \\
\text { Anticipada } \\
\left(\mathbf{i}_{\mathrm{a}}\right)\end{array}$} & Nominal Aniticipada & $J_{a}=i_{a}^{*} m$ & $J_{a}=\left(1-\left(1-i_{a}\right)^{(m / m d)}\right)^{*} m d$ \\
\hline & Nominal Vencida & $J=\left(1 /\left(1-i_{a}\right)-1\right)^{*} m$ & $J=\left(1 /\left(1-i_{a}\right)^{(m / m d)}-1\right)^{*} m d$ \\
\hline & Efectiva Anicicipada & & $\mathrm{i}_{\mathrm{a}}=1-\left(1-\mathrm{i}_{\mathrm{a}}\right)^{(\mathrm{m} / \mathrm{md})}$ \\
\hline & \begin{tabular}{|l} 
Efectiva Vencida \\
\end{tabular} & $\mathrm{i}=\mathrm{i}_{\mathrm{a}} /\left(1-\mathrm{i}_{\mathrm{a}}\right)$ & $\mathrm{i}=1 /\left(1-\mathrm{i}_{\mathrm{a}}\right)^{(\mathrm{m} / \mathrm{md})}-1$ \\
\hline \multirow{4}{*}{$\begin{array}{c}\text { Efectiva } \\
\text { Vencida } \\
\text { (i) }\end{array}$} & Nominal Anicipipada & $J_{a}=(1-1 /(1+i))^{*} m$ & $J_{a}=\left(1-1 /(1+i)^{(m / m d)}\right)^{*} m d$ \\
\hline & Nominal Vencida & $\mathrm{J}=\mathrm{i}^{*} \mathrm{~m}$ & $J=\left((1+i)^{(m / m d)}-1\right)^{*} m d$ \\
\hline & Eféctiva Anicipipada & $i_{a}=i /(1+i)$ & $i_{a}=1-1 /(1+i)^{(m / m d)}$ \\
\hline & Efectiva Vencida & & $i=(1+i .)^{(m / m d)}-1$ \\
\hline
\end{tabular}

Fuente: Elaboración profesor Milton Triana.

La tercera columna muestra las fórmulas a emplear solo si la conversión que se efectúa cambia únicamente la clase y/o la modalidad, pero no la periodicidad; nótese que hay solo 3 opciones de destino.

Y la cuarta columna muestra las fórmulas a emplear cuando la conversión implica un cambio tanto de la clase y/o la modalidad como de la periodicidad; nótese aquí que hay 4 opciones de destino posibles.

Recapitulando, el paso 1 y el paso 2 por la columna 1 por la columna 2 , respectivamente, son sucesivos y obligatorios, en tanto que el paso 3 y el paso 4 son excluyentes, porque puede se puede pasar a la columna 3 , si se cambia la clase y/o la modalidad, pero no la periodicidad, o a la columna 4, si se cambia la clase y/o la modalidad junto con la periodicidad.

Finalmente, se precisa el manejo de las siguientes convenciones para entender y aplicar las fórmulas previstas en la columna 3 o en la columna 4:
J: Tasa de interés nominal vencida

$\mathbf{J}_{\mathbf{a}}$ : Tasa de interés nominal anticipada

i: Tasa de interés efectiva vencida

$\mathbf{i}_{\mathbf{a}}$ : Tasa de interés efectiva anticipada

m: Periodicidad conocida

md: Periodicidad desconocida

\section{Contraste de los procedimientos tradicionales contra la "Matriz MILHER" para convertir tasas de interés financiero.}

Inicialmente, se realizó un recuento explicativo de los cinco procedimientos utilizados para convertir tasas de interés: la Gráfica de equivalencia de tasas de Baca Currea, el Con base en preguntas de Ramírez y Martínez, el Diagrama de conversión de tasas de interés de Moreno y Rueda, el de Transformación de tasas de interés de Vélez y la Gráfica de conversión de tasas de interés de Meza Orozco.

Luego, se aplicó la Encuesta 01 con estudiantes, de los cuales el 53,06\% eran de la Corporación universitaria Minuto de Dios, UNIMINUTO, y el restante 46,94\% eran de la Universidad Santo Tomás, USTA, la cual contenía dos preguntas de selección múltiple con única respuesta.

En cuanto al reconocimiento de los elementos de una tasa de interés financiero, en su orden reconocen más fácilmente la periodicidad (un 93.88\%), luego la modalidad (un 89.80\%) y por último la clase (un 87.76\%), se puede apreciar en la Tabla 1. 
Tabla 1: Pregunta 1: Cuando se le presenta una tasa de interés...

\begin{tabular}{|c|c|c|c|}
\hline$X_{i}$ & & $\mathrm{n}_{\mathrm{i}}$ & $\mathbf{h}_{\mathrm{i}}$ \\
\hline \multirow{2}{*}{ 1.1. Reconoce la clase } & $\mathrm{Si}$ & 43 & 0,8776 \\
\hline & No & 6 & 0,1224 \\
\hline \multirow{2}{*}{ 1.2. Reconoce la periodicidad } & $\mathrm{Si}$ & 46 & 0,9388 \\
\hline & No & 3 & 0,0612 \\
\hline 1.3. Reconoce la modalidad & $\begin{array}{l}\mathrm{Si} \\
\mathrm{No}\end{array}$ & $\begin{array}{c}44 \\
5\end{array}$ & $\begin{array}{l}0,8980 \\
0,1020\end{array}$ \\
\hline
\end{tabular}

Fuente: Elaboración autor

Por otro lado, en cuanto al método o procedimiento utilizado para convertir tasas de interés financiero (Figura 7), un 46.94\% expresó que aplica el método de igualación "Con base en preguntas", un 40.82\% manifiesta que aplica la "Gráfica de equivalencia de tasas" y un 12.24\% mencionó "No sabe no responde", muy a pesar que se hizo referencia (al inicio del abordaje) a estos métodos o procedimientos de conversión de tasas, aunque cabe decir que hubo tres métodos o procedimientos que no son utilizados o seguidos por estudiantes y docentes.

Figura 7. Pregunta 2: Procedimiento utilizado para convertir tasas de interés

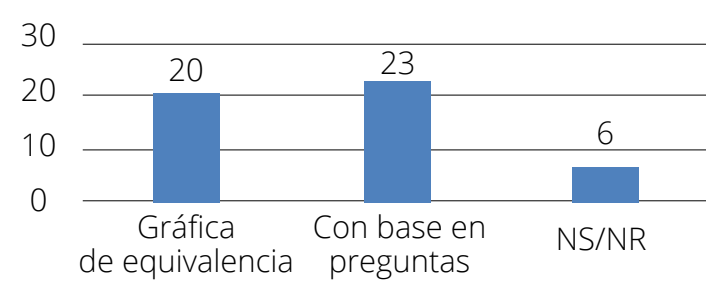

Fuente: Elaboración autor

Posteriormente, se operaron dos ejercicios de prueba para verificar su correcta aplicación y luego se procedió a proponer otros ejercicios similares a los propuestos, en diferente orden para evitar favorabilidad en este nuevo escenario.
El primer ejercicio implicaba cambiar la clase, la periodicidad y la modalidad; el segundo ejercicio implicaba cambiar la periodicidad, mas no la clase y la modalidad; el tercero implicaba cambiar la periodicidad y la modalidad, mas no la clase; y el cuarto ejercicios implicaba cambiar la periodicidad, mas no la clase y la modalidad.

\section{Discusión Ejercicio 1}

\section{Solución con la "Gráfica de equivalencia de tasas" de Baca Currea}

"De una tasa del 17\% N.B.V. hallar una tasa efectiva trimestral anticipada."

Inicialmente, se cambia la clase:

$\mathbf{i}=\mathbf{J} / \mathbf{m} \longrightarrow \mathrm{i}=0,17 / 6=0,028333333$ E.B.V.

Luego, se cambia la periodicidad:

$\mathrm{i}=\left(1+\mathrm{i}_{\mathrm{c}}\right)^{(\mathrm{mc} / \mathrm{md})}-1 \rightarrow \mathrm{i}=(1+0,028333333)^{(6 / 4)}-1=0,042799635$ E.T.V.

Por último, se cambia la modalidad:

$\mathrm{i}_{\mathrm{a}}=\mathrm{i} /(1+\mathrm{i}) \rightarrow \mathrm{i}_{\mathrm{d}}=0,042790635 /(1+0,042790635)=0,041043009 \quad$ E.T.A.

La respuesta final:

$$
i_{\mathrm{a}}=0,0410430092 \text { E.T.A. }
$$

\section{Solución "Con base en preguntas" de Ramírez y Martínez}

"De una tasa del 17\% N.B.V. hallar una tasa efectiva trimestral anticipada."

Se plantea la igualdad: de izquierda a derecha antes del igual se ubican los datos de la tasa conocida (la que se va 
a convertir) y después del igual se ubican los datos de la tasa desconocida.

En el primer paréntesis el signo después de la constante es "más", porque la modalidad de la tasa conocida es vencida; como su tasa es nominal se indica la división del factor decimal entre la periodicidad de la tasa conocida; $y$, finalmente, el exponente es positivo porque la modalidad es vencida.

En el segundo paréntesis el signo después de la constante es "menos" porque la modalidad de la tasa desconocida es anticipada; como su tasa es efectiva se deja indicada la incógnita "i"; y, finalmente, el exponente es negativo porque la modalidad es anticipada.

$$
\left(1+\frac{0,17}{6}\right)^{6}=(1-i)^{-4}
$$

Se va dando solución, dividiendo ambos exponentes por el mismo valor del exponente que está después de igual. Si se estima conveniente se halla el resultado dentro del primer paréntesis antes del igual y si aplica, se pueden dividir los exponentes, teniendo en cuenta la ley de los signos.

$$
\begin{gathered}
(1+0,0283)^{6 /-4}=(1-\mathrm{i}) \\
1,0283^{-1,5}=1-\mathrm{i}
\end{gathered}
$$

Se sigue despejando según convenga:

$$
\mathrm{i}=1-0,9589569908
$$

La respuesta final:

$$
i_{\mathrm{a}}=0,0410430092 \text { E.T.A. }
$$

\section{Solución con la “Matriz MILHER"}

"De una tasa del 17\% N.B.V. hallar una tasa efectiva trimestral anticipada."

Se ubica la tasa conocida en la columna 1 (segunda caja, es una tasa nominal vencida); luego se ubica al frente de la caja seleccionada el renglón correspondiente de la tasa desconocida en la columna 2 (primer renglón, es una tasa nominal anticipada); seguidamente se toma la decisión de buscar y utilizar la fórmula correspondiente en la columna 4 (porque la tasa desconocida tiene diferente periodicidad) por el mismo renglón ubicado en la columna 2 (primer renglón, es una tasa anticipada).

Así pues, la fórmula es:

$$
\mathbf{i}_{\mathbf{a}}=1-(\mathbf{1}+\mathbf{J} / \mathbf{m})^{(-\mathbf{m} / \mathbf{m d})}
$$

Se ubican las variables respectivas al operar la fórmula:

$i_{a}=1-(1+0,17 / 6)^{(-6 / 4)}=0,0410430092$ E.T.A.

La respuesta final:

$$
i_{\mathrm{a}}=0,0410430092 \text { E.T.A. }
$$

\section{Discusión Ejercicio 2}

\section{Solución con la "Gráfica de equivalencia de tasas" de Baca Currea}

"Convertir una tasa del 28\% N.S.V. a una tasa nominal mensual vencida".

Inicialmente, se cambia la clase:

$$
\mathrm{i}=0,28 / 2=0,14 \text { E.S.V. }
$$


Novedasa herramienta didáctica para la conversión de tasas de interés financiero, 49-69

Luego, se cambia la periodicidad:

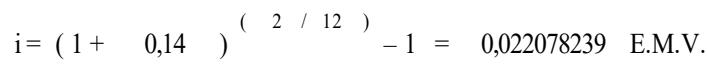

Finalmente, se cambia nuevamente la clase:

$\mathrm{J}=0,022078239 * 12=0,2649388691 \quad$ N.M.V

La respuesta final:

$$
\mathbf{J}=\text { 0,2649388691 N.M.V. }
$$

\section{Solución "Con base en preguntas" de Ramírez y Martínez}

"Convertir una tasa del 28\% N.S.V. a una tasa nominal mensual vencida."

Se plantea la igualdad: de izquierda a derecha antes del igual se ubican los datos de la tasa conocida (la que se va a convertir) y después del igual se ubican los datos de la tasa desconocida.

En el primer paréntesis el signo después de la constante es "más" porque la modalidad de la tasa conocida es vencida; como su tasa es nominal se indica la división del factor decimal entre la periodicidad de la tasa conocida; $y$, finalmente, el exponente es positivo porque la modalidad de la tasa conocida es vencida.

En el segundo paréntesis el signo después de la constante es "más" porque la modalidad de la tasa desconocida es vencida; como su tasa es nominal se deja indicada la división de la incógnita "i" entre la periodicidad de la tasa desconocida; $y$, finalmente, el exponente es positivo porque la modalidad de la tasa conocida es vencida.

$$
\left(1+\frac{0,28}{2}\right)^{2}=\left(1+\frac{\mathrm{i}}{12}\right)^{12}
$$

Se va dando solución, dividiendo ambos exponentes por el mismo valor del exponente que está después de igual. Si se estima conveniente se halla el resultado dentro del primer paréntesis antes del igual y si aplica se pueden dividir los exponentes, teniendo en cuenta la ley de los signos.

$$
(1+0,1400)^{2 / 12}=\left(1+\frac{\mathrm{i}}{12}\right)
$$

Se puede resolver antes del igual y luego se traspone según convenga:

$$
1,1400^{0,16667}=1+\frac{\mathrm{i}}{12}
$$

Se sigue despejando según convenga:

$$
\begin{gathered}
\frac{\mathrm{i}}{12}=1,022078239-1 \\
\frac{\mathrm{i}}{12}=0,022078239 \\
\mathrm{i}=0,022078239 * 12
\end{gathered}
$$

La respuesta final:

$$
\mathbf{J}=\text { 0,2649388691 N.M.V. }
$$

\section{Solución con la "Matriz MILHER"}

"Convertir una tasa del 28\% N.S.V. a una tasa nominal mensual vencida."

Se ubica la tasa conocida en la columna 1 la segunda caja (tasa nominal vencida); luego se elige, frente de la caja seleccionada el renglón de la tasa desconocida en la columna 2 (tasa nominal vencida); seguidamente se toma la decisión de utilizar la fórmula correspondiente en la columna 
4 (porque la tasa desconocida tiene diferente periodicidad) por el mismo renglón ubicado en la columna 2.

Así pues, la fórmula que se usa es:

$$
\mathbf{J}=\left((\mathbf{1}+\mathbf{J} / \mathbf{m})^{(\mathbf{m} / \mathbf{m d})}-1\right) * \mathbf{m d}
$$

Se ubican las variables respectivas al desarrollar la fórmula:

$\mathrm{J}=\left((1+0,28 / 2)^{(2 / 12)}-1\right) * 12=0,2649388691$ N.T.V.

La respuesta final:

\begin{tabular}{|ll|}
\hline$J=0,2649388691$ & N.M.V. \\
\hline
\end{tabular}

\section{Discusión Ejercicio 3}

\section{Solución con la "Gráfica de equivalencia de tasas" de Baca Currea}

"Hallar una tasa nominal trimestral anticipada de una tasa del 32\% N.B.V."

Inicialmente, se cambia la clase:

$$
\mathrm{i}=0,32 / 6=0,05333 \text { E.B.V. }
$$

Se cambia la periodicidad:

$$
i=(1+0,05333)^{(6 / 4)}-1=0,081057370 \text { E.T.V. }
$$

Luego, la modalidad

$i_{a}=0,08105737 /(1+0,08105737)=0,074979712 \quad$ E.T.A.

Y nuevamente la clase:

$\mathrm{J}_{\mathrm{a}}=0,074979712 * 4=0,299918847$ N.T.A.

La respuesta final:

$$
\mathbf{J}_{\mathrm{a}}=\mathbf{0 , 2 9 9 9 1 8 8 4 7 \text { N.T.A. }}
$$

\section{Solución "Con base en preguntas" de Ramírez y Martínez}

"Hallar una tasa nominal trimestral anticipada de una tasa del 32\% N.B.V."

Se plantea la igualdad: de izquierda a derecha antes del igual se ubican los datos de la tasa conocida (la que se va a convertir) y después del igual se ubican los datos de la tasa desconocida.

En el primer paréntesis el signo después de la constante es "más" porque la modalidad de la tasa conocida es vencida; como su tasa es nominal se indica la división del factor decimal entre la periodicidad de la tasa conocida; $y$, finalmente, el exponente es positivo porque la modalidad de la tasa conocida es vencida.

En el segundo paréntesis el signo después de la constante es "menos" porque la modalidad de la tasa desconocida es anticipada; como su tasa es nominal se deja indicada la división de la incógnita "i" entre la periodicidad de la tasa desconocida; y, finalmente, el exponente es negativo porque la modalidad de la tasa conocida es anticipada.

$$
\left(1+\frac{0,32}{6}\right)^{6}=\left(1-\frac{\mathrm{i}}{4}\right)^{-4}
$$

Se va dando solución, dividiendo ambos exponentes por el mismo valor del exponente que está después de igual. Si se estima conveniente se halla el resultado dentro del primer paréntesis antes del igual y si aplica se pueden dividir los exponentes, teniendo en cuenta la ley de los signos. 
Se puede resolver antes del igual y luego se traspone según convenga:

$$
1,0533^{-} 1,5=\left(1-\frac{\mathrm{i}}{4}\right)
$$

Se sigue despejando según convenga:

$$
\begin{gathered}
\frac{\mathrm{i}}{4}=1-0,92502028832 \\
\frac{\mathrm{i}}{4}=0,074979712 \\
\mathrm{i}=0,074979712 * 4
\end{gathered}
$$

La respuesta final:

$$
\mathbf{J}_{\mathrm{a}}=\mathbf{0 , 2 9 9 9 1 8 8 4 7 \text { N.T.A. }}
$$

\section{Solución con la “Matriz MILHER"}

Hallar una tasa nominal trimestral anticipada de una tasa del 32\% N.B.V.

Se ubica la tasa conocida en la columna 1 la segunda caja (tasa nominal vencida); luego se elige el renglón correspondiente de la tasa desconocida en la columna 2 (tasa nominal anticipada); seguidamente se toma la decisión de utilizar la fórmula correspondiente en la columna 4 (porque la tasa desconocida tiene diferente periodicidad) por el mismo renglón ubicado en la columna 2 (tasa nominal anticipada).

Así pues, la fórmula a aplicar es:

$$
\mathbf{J}_{\mathrm{a}}=\left(\mathbf{1}-(\mathbf{1}+\mathbf{J} / \mathbf{m})^{(-\mathbf{m} / \mathbf{m d})}\right) * \mathbf{m d}
$$

Se ubican las variables respectivas al desarrollar la fórmula:
$\mathrm{J}_{\mathrm{a}}=\left(1-(1+0,32 / 6)^{(-6 / 4)}\right) * 4=0,2999188467$ N.T.A. La respuesta final:

$$
\mathrm{J}_{\mathrm{a}}=\mathbf{0 , 2 9 9 9 1 8 8 4 7 \text { N.T.A. }}
$$

\section{Discusión Ejercicio 4}

\section{Solución con la "Gráfica de equivalencia de tasas" de Baca Currea}

"Con una tasa del 25\% N.M.A. hallar una tasa nominal semestral anticipada equivalente."

Inicialmente se cambia la clase:

$$
\mathrm{i}_{\mathrm{a}}=0,25 / 12=0,020833333 \text { E.M.A }
$$

Seguidamente la modalidad

$i=0,020833333 /(1-0,020833333)=0,02127659574$ E.M.V.

Luego la periodicidad:

$\mathrm{i}=(1+0,02127659574)^{(12 / 2)}-1=0,1346457131$ E.S.V.

Nuevamente la modalidad

$\mathrm{i}_{\mathrm{a}}=0,1346457131 /(1+0,1346457131)=0,1186676260$ E.S.A.

Finalmente, se cambia otra vez la clase:

$\mathrm{J}_{\mathrm{a}}=0,1186676260 * 2=0,2373352520 \quad$ N.S.A.

La respuesta final:

$$
\mathbf{J}_{\mathrm{a}}=0,2373352520 \text { N.S.A. }
$$




\section{Solución "Con base en preguntas" de Ramírez y Martínez}

"Con una tasa del 25\% N.M.A. hallar una tasa N.S.A. equivalente."

Se plantea la igualdad: de izquierda a derecha antes del igual se ubican los datos de la tasa conocida (la que se va a convertir) y después del igual se ubican los datos de la tasa desconocida.

En el primer paréntesis el signo después de la constante es "menos" porque la modalidad de la tasa conocida es anticipada; como su tasa es nominal se indica la división del factor decimal entre la periodicidad de la tasa conocida; $y$, finalmente, el exponente es negativo porque la modalidad de la tasa conocida es anticipada.

En el segundo paréntesis el signo después de la constante es "menos" porque la modalidad de la tasa desconocida es anticipada; como su tasa es nominal se deja indicada la división de la incógnita "i" entre la periodicidad de la tasa desconocida; $y$, finalmente, el exponente es negativo porque la modalidad de la tasa conocida es anticipada.

$$
\left(\mathrm{i}-\frac{0.25}{\mathrm{i} 2}\right)^{-12}=\left(\mathrm{i}-\frac{1}{2}\right)^{-2}
$$

Se va dando solución, dividiendo ambos exponentes por el mismo valor del exponente que está después de igual. Si se estima conveniente se halla el resultado dentro del primer paréntesis antes del igual y si aplica se pueden dividir los exponentes, teniendo en cuenta la ley de los signos.
Se puede resolver antes del igual y luego se traspone según convenga:

$$
0,9792^{6}=\left(\begin{array}{ll}
1-\frac{\mathrm{i}}{2}
\end{array}\right)
$$

Se sigue despejando según convenga:

$$
\begin{aligned}
& \frac{\mathrm{i}}{2}=1-0,8813323740 \\
& \frac{\mathrm{i}}{2}=0,1186676260 \\
& \mathrm{i}=0,1186676260 * 2
\end{aligned}
$$

La respuesta final:

$$
\mathbf{J}_{\mathbf{a}}=\mathbf{0 , 2 3 7 3 3 5 2 5 2 0} \text { N.S.A. }
$$

\section{Solución con la "Matriz MILHER"}

"Con una tasa del 25\% N.M.A. hallar una tasa nominal semestral anticipada equivalente."

Se ubica la tasa conocida en la columna 1 (tasa nominal anticipada); luego se elige el renglón correspondiente de la tasa desconocida en la columna 2 (tasa nominal anticipada); seguidamente se toma la decisión de utilizar la fórmula correspondiente en la columna 4 (porque la tasa desconocida tiene diferente periodicidad) por el mismo renglón ubicado en la columna 2 (primer renglón, es una tasa nominal anticipada).

Así pues, se emplea la siguiente fórmula:

$$
(1-0,0208)^{-12 /-2}=\left(1-\frac{\mathrm{i}}{2}\right)
$$$$
J_{a}=\left(1-\left(1-J_{a} / m\right)^{(m / m d)}\right) * m d
$$ 
Se ubican las variables respectivas al desarrollar la fórmula:

$\mathrm{J}_{\mathrm{a}}=\left(1-(1-0,25 / 12)^{(12 / 2)}\right) * 2=0,2373352520$ N.S.A.

La respuesta final:

$$
\mathbf{J}_{\mathrm{a}}=0,2373352520 \text { N.S.A. }
$$

\section{Discusión final}

Cabe destacar, inicialmente, que los resultados en las mediciones del tiempo utilizado fueron interesantes (Figura 8).

Una primera novedad es que la "Matriz MILHER" (en su segunda versión), puede seguir mejorándose y continúa manejando la aplicación de una fórmula y se hace tan solo un cálculo, en tanto que con el procedimiento que usa la "Gráfica de equivalencia de tasas" de Baca Currea se pueden realizar los cálculos con la utilización de una hasta cinco fórmulas consecutivas, o con el procedimiento que soluciona "Con base en preguntas" de Ramírez y Martínez se pueden ejecutar desde dos hasta cuatro o cinco cálculos, dependiendo de la destreza matemática del usuario en los despejes que se lleven a cabo.

Aunado a lo anterior, hay un ahorro promedio de tiempo en su utilización del 36\% respecto al procedimiento que soluciona "Con base en preguntas" de Ramírez y Martínez, y del 79\% respecto al procedimiento que usa la "Gráfica de equivalencia de tasas" de Baca Currea, lo cual convierte a la "Matriz MILHER" en una herramienta bastante eficiente y muy práctica al momento de su empleo.
Figura 8. Tiempo de solución en segundos de los 4 ejercicios propuestos

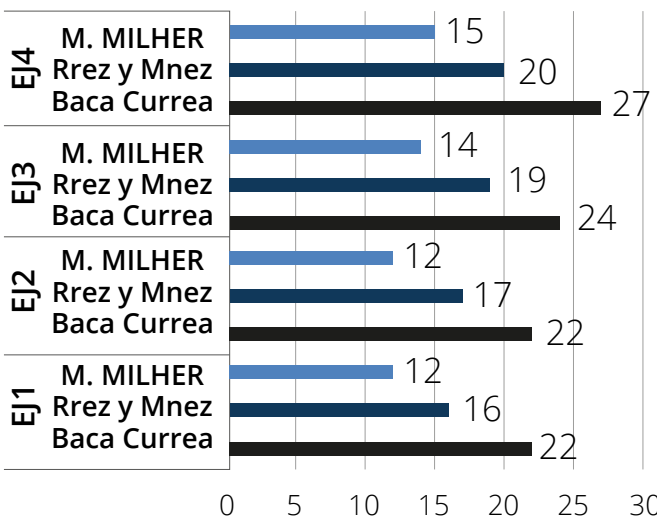

Fuente: Elaboración autor.

Finalmente, hubo una muy buena percepción en cuanto a la explicación necesaria para poder aplicar la "Matriz MILHER" (Figura 9) y en cuanto al hecho de si la herramienta facilita aun más el proceso de conversión de tasas (Figura 10).

Figura 9. Pregunta1: Información para aplicar la Matriz MILHER

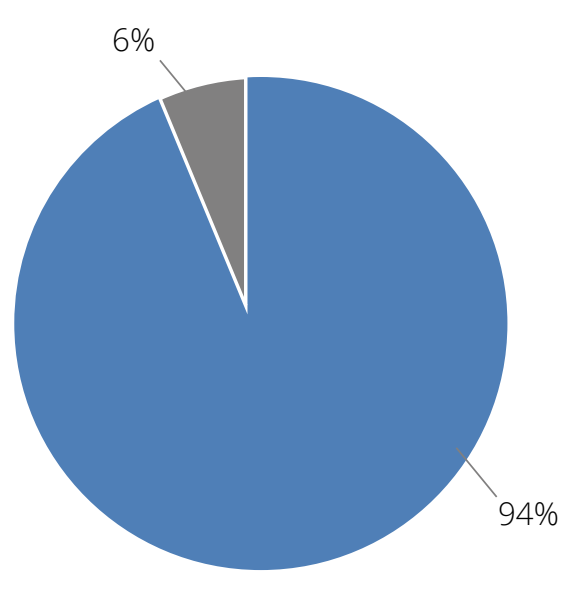

Sí No

Fuente: Elaboración autor 
Figura 10. Pregunta 2: Aplicabilidad de la Matriz MILHER

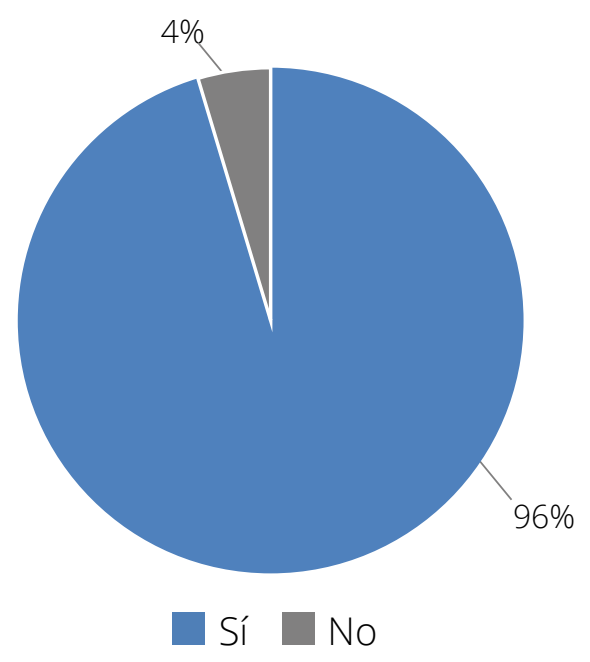

Fuente: Elaboración autor.

Así pues, la inducción inicial donde se explicó la mecánica de la "Matriz MILHER" fue necesaria y lo suficientemente amplia, lo cual facilitó el proceso de operatividad para la conversión de tasas de interés, que se comprobó con la consecución de los resultados obtenidos y contrastados; las críticas fueron también positivas y favorables, hubo aportes por parte de los estudiantes, que se dieron en dos direcciones: el primero, que se colocara en la parte inferior las periodicidades, y el segundo, que se ubicara un instructivo leve sobre su manejo con 4 instrucciones sobre la aplicación de la novedosa herramienta.

Igualmente, la "Matriz MILHER" es funcional y amigable con su usuario, desde el punto de vista de los estudiantes tuvo bastante aceptación, tal como se comprobó en los dos escenarios de esta prueba: la Corporación Universitaria Minuto de Dios, UNIMINUTO, y la Universidad Santo Tomás,
USTA, ambas instituciones de educación superior ubicadas en Villavicencio (Meta).

\section{Conclusiones}

Al explicar la estructura y el funcionamiento de la Matriz MILHER (en su segunda versión) se reafirma que la conversión de tasas de interés financiero es un tema "muy importante" para la comprensión los temas subsecuentes de la asignatura de matemáticas financieras para estudiantes y, posteriormente, para profesionales en ejercicio.

Por otro lado, al contrastar experimentalmente la herramienta didáctica, Ilamada la Matriz MILHER, con otros procedimientos empleados para la conversión de tasas de interés, se demostró que es mejor en términos de eficiencia (economiza el tiempo de uso) y de aplicabilidad (menor complejidad, simplifica el proceso), lo cual facilita efectivamente los procesos de aprendizaje y de enseñanza.

Finalmente, se espera replicar la novedosa herramienta, no solo en aulas de clase sino en otros escenarios como en la web, de tal forma que con un video explicativo u otro formato de la Matriz MILHER tipo app en dispositivos móviles y/o favorecer la implementación de "sistemas de aprendizaje virtual conocidos como e-learning, que ayudarían en forma asincrónica el crecimiento de los colaboradores" en las empresas (Torres Flórez, 2019, pág. 8) y a los aun estudiantes en proceso de formación de universidades, de tal forma que se pueda conseguir el objetivo de con- 
vertir tasas de interés financiero en cualquier momento de una forma práctica y amigable, y ahorrando preciado tiempo en su operación.

\section{Referencias}

Agudelo Fernández, D. A. (2019). Las matemáticas financieras. Conceptos y aplicaciones. Bogotá D.C.: Editorial Pearson Educación.

Baca Currea, G. (2007). Ingeniería Económica. Bogotá D.C.: Fondo Educativo Panamericano.

Blank, L., \& Tarquin, A. (2006). Ingeniería económica. México D.F.: Mc Graw Hill Interamericana Editores S.A. de C.V.

Buenaventura Vera, G. (2018). Fundamentos de matemáticas financiera. Bogotá D.C.: ECOE Ediciones.

Cano Morales, A. M. (2017). Matemáticas financieras, aplicado a ciencias económicas, administrativas y contables. Bogotá D.C.: Ediciones de la U.

Carrasco Manchado, A. I., \& Rábade Obradó, M. I. (2008). Pecar en la Edad Media. Madrid (España): Silex Ediciones. Obtenido de https:// books.google.com.co/books?id=iFUWdwjGeDMC\&Ipg =PA 193\&d$q=e l \% 20$ inter $\%$ C3\%A9s $\% 20$ de $\% 20$ los\%20pr\%C3\%A9stamos\%20en\%20 la\%20edad\%20antigua\&hl=es\&p$\mathrm{g}=\mathrm{PA} 193 \# \mathrm{v}=$ onepage $\& \mathrm{q}=\mathrm{el} \% 20$ inter\%C3\%A9s\%20de\%20los\%20 pr\%C3\%A9stamos\%20en\%20la\%20 edad\%20antigua\&f=false

Diaz Mata, A., \& Aguilera Gómez, V. M. (2013). Matemáticas financieras. México D.F.: Mc Graw Hill Interamericana Editores S.A. de C.V.

Frare, M. J. (2005). La generación del interés desde el modelo matemático.
Revista de la Facultad de ciencias económicas, 82-101.

Gutiérrez Carmona, J. (2012). Matemáticas financieras. Bogotá D.C.: ECOE Ediciones.

Hernandez Sampieri, R., Fernández Collado, C., \& Baptista Lucio, M. d. (2010). Metodología de la investigación. México D.F.: McGraw Hill / Interamericana Editores S.A. de C.V.

Herrera Aráuz, D. (2017). Matemática financiera. Bogotá D.C.: Alfaomega Colombiana S.A.

Jiménez Muñoz, F. J. (2010). La Usura. Evolución histórica y patología de los intereses. Madrid (España): Dikynson S.L. Obtenido de https:// books.google.com.co/books?i$\mathrm{d}=\mathrm{g}_{-}$Tb3ugx8jQC\&lpg=PA32\&d$q=e l \% 20$ inter $\%$ C3\%A9s\%20de\%20 los\%20pr\%C3\%A9stamos\%20en\%20 la\%20edad\%20moderna\&hl=es\&p$\mathrm{g}=\mathrm{PA} 42 \# \mathrm{v}=$ onepage $\& \mathrm{q}=\mathrm{el} \% 20 \mathrm{in}$ ter $\%$ C 3\%A 9s $\% 20$ de $\% 20$ los $\% 20$ pr\%C3\%A9stamos\%20en\%20la\%20 edad\%20moderna\&f=false

Lezama Vásquez, J. (2006). Matemáticas financieras II. Chimbote (Perú): Sistema de Educación Abierta - ULADECH.

Margaria, O., \& Bravino, L. (2014). Matemática financiera. Montería (Colombia): Universidad Nacional de Córdoba.

Meza Orozco, J. d. (2017). Matemáticas financieras aplicadas. Bogotá D.C.: ECOE Ediciones.

Mira Navarro, J. C. (2019). Introducción a las operaciones financieras. Madrid (España).

Morales Castaño, C. M. (2014). Finanzas del Proyecto. Introducción a las matemáticas financieras. Medellín Colombia: Centro Editorial Esumer. 
Moreno Gómez, N. E., \& Rueda Forero, P. (1998). Matemáticas financieras. Bucaramanga (Colombia): Universidad Industrial de Santander, UIS.

Pérez Paredes, A., Cruz de los Ángeles, J. A., \& Gómez Pulido, A. M. (2018). Situación actual de la Educación financiera en los Jóvenes Universitarios de Villavicencio Colombia. Revista GEON, 5(2), 115-130. Obtenido de http:// revistageon.unillanos.edu.co/index. php/geon/article/view/81/70

Ramírez Mora, J. M., \& Martínez Cárdenas, É. E. (2016). Matemática financiera. Interés, tasas y equivalencias. Bogotá D.C.: Editorial TRILLAS de CoIombia Ltda.

Reichardt, C. S., \& Cook, T. D. (1986). Métodos cualtitativos y cuantitativos en investigación evaluativa. Madrid: Ediciones Morata, S. L.

Rosillo C., J. S. (2009). Matemáticas financieras para decisiones de inversión y financiación. Bogotá D.C.: Cengage Learning Editores S.A. de C. V.

Torres Flórez, D. (2019). El entrenamiento del colaborador como estrategia de mejoramiento continuo. GEON Revista, 6(1), 4-9. Obtenido de http:// revistageon.unillanos.edu.co/index. php/geon/article/view/151/143

Vélez Pareja, I. (2006). Decisiones de inversión para valoración financiera de proyectos y empresas. Bogotá D.C.: Pontificia Universidad Javeriana.

Villalobos Pérez, J. L. (2017). Matemáticas financieras. Bogotá D.C.: Editorial Pearson Educación. 